\title{
Electroacupuncture Pretreatment Attenuates Cerebral Ischemic Injury via Notch Pathway-Mediated Up-Regulation of Hypoxia Inducible Factor-1 $\alpha$ in Rats
}

\author{
Yu Zhao ${ }^{1,2} \cdot$ Bin Deng ${ }^{1} \cdot{\text { Yichong } \mathrm{Li}^{2} \cdot \text { Lihua } \mathrm{Zhou}^{2} \cdot \text { Lei Yang }}^{4} \cdot \mathrm{Xingchun} \mathrm{Gou}^{3}$ • \\ Qiang Wang ${ }^{4} \cdot$ Guozhong Chen ${ }^{5} \cdot \mathrm{Hao} \mathrm{Xu}^{3} \cdot$ Lixian $\mathrm{Xu}^{1}$
}

Received: 16 February 2015 / Accepted: 29 April 2015/Published online: 15 May 2015

(c) The Author(s) 2015. This article is published with open access at Springerlink.com

\begin{abstract}
We have reported electroacupuncture (EA) pretreatment induced the tolerance against focal cerebral ischemia through activation of canonical Notch pathway. However, the underlying mechanisms have not been fully understood. Evidences suggest that up-regulation of hypoxia inducible factor- $1 \alpha$ (HIF-1 $\alpha$ ) contributes to neuroprotection against ischemia which could interact with Notch signaling pathway in this process. Therefore, the current study is to test that up-regulation of HIF- $1 \alpha$ associated with Notch pathway contributes to the neuroprotection of EA pretreatment. Sprague-Dawley rats were treated with EA at the acupoint "Baihui (GV 20)" $30 \mathrm{~min}$ per day for successive 5 days before MCAO. HIF- $1 \alpha$ levels were measured before and after reperfusion. Then, HIF- $1 \alpha$ antagonist 2ME2 and $\gamma$-secretase inhibitor MW167 were used. Neurologic deficit scores, infarction volumes, neuronal apoptosis, and Bcl2/Bax were evaluated. HIF- $1 \alpha$ and
\end{abstract}

Yu Zhao and Bin Deng have contributed equally to this work.

Hao Xu

haoxu83@hotmail.com

$\triangle$ Lixian Xu

xlx116@fmmu.edu.cn

1 State Key Laboratory of Military Stomatology, Department of Anesthesiology, School of Stomatology, The Fourth Military Medical University, Xi'an 710032, China

2 Department of Anesthesiology, Binghua Hospital, Haerbin 150080, China

3 Institution of Basic Medical Science, Xi'an Medical University, Xi'an 710032, China

4 Department of Anesthesiology, Xijing Hospital, Fourth Military Medical University, Xi' an 710032, China

5 Department of Anesthesiology, Fuzhou General Hospital, Fuzhou 350015, China
Notch1 intracellular domain (NICD) were assessed. The results showed EA pretreatment enhanced the neuronal expression of HIF- $1 \alpha$, reduced infarct volume, improved neurological outcome, inhibited neuronal apoptosis, upregulated expression of $\mathrm{Bcl}-2$, and down-regulated expression of Bax after reperfusion in the penumbra, while the beneficial effects were attenuated by 2 ME2. Furthermore, intraventricular injection with MW167 efficiently suppressed both up-regulation of NICD and HIF-1 $\alpha$ after reperfusion. However, administration with $2 \mathrm{ME} 2$ could only decrease the expression of HIF- $1 \alpha$ in the penumbra. In conclusion, EA pretreatment exerts neuroprotection against ischemic injury through Notch pathway-mediated upregulation of HIF- $1 \alpha$.

Keywords HIF-1 $\alpha$ - Notch pathway $\cdot$ Stroke $\cdot$ Penumbra . Electroacupuncture $\cdot$ Neurobehavior function $\cdot$ Apoptosis

\section{Introduction}

Stroke is a major cause of death and disability in adults throughout the world, and only a minority of stroke patients receives thrombolytic therapy due to the narrow time window and side effects associated with the thrombolytic agent (Go et al. 2013). Therefore, new strategies focusing on neuroprotection are urgently needed. Preconditioning, as a potent endogenous protective procedure, activates several endogenous signaling pathways that protect against ischemia (Wang et al. 2009). Electroacupuncture (EA) has been shown to produce clinically beneficial effects in stroke patients, and EA pretreatment can also induce tolerance against ischemia (Zhao et al. 2012). However, the signaling mechanisms mediating the effects of EA pretreatment are unclear. 
HIF- $1 \alpha$ is a transcription factor that plays a key role in regulating the adaptive response to hypoxia by regulating the expression of its target genes, which include heme oxygenase1 (HO-1) (Singh et al. 2012). HIF-1 $\alpha$ has been shown to protect neurons from death during the initial $24 \mathrm{~h}$ following mild hypoxia (Lopez-Hernandez et al. 2012). Previous studies have shown that the neuroprotection against focal cerebral ischemia provided by various agents is through the upregulation of HIF-1 $\alpha$ (Wang et al. 2012a; Yuan et al. 2011; Doeppner et al. 2012). Whether HIF-1 $\alpha$ acts in a similar manner after EA neuroprotective pretreatment is unclear.

Our previous study demonstrated that EA pretreatmentinduced tolerance against cerebral ischemia by activating the canonical Notch signaling pathway. A potential link between HIF-1 $\alpha$ and Notch signaling has been reported (Wilkins et al. 2009). Additionally, the expression of HIF$1 \alpha$ and its downstream target genes requires the phosphorylation of signal transducer and activator of transcription 3 (STAT3), and Notch signaling can activate STAT3 phosphorylation (Jung et al. 2005; Kamakura et al. 2004). Notch and HIF-1 $\alpha$ appear to share several levels of molecular regulatory mechanisms.

Therefore, the aims of the current study are to test the hypothesis that the up-regulation of HIF-1 $\alpha$ contributes to the neuroprotective effects of EA pretreatment and to determine whether the neuroprotection induced by the upregulation of HIF- $1 \alpha$ is associated with the Notch signaling pathway.

\section{Materials and Methods}

\section{Experimental Protocols}

\section{Experiment I}

To assess the effect of EA pretreatment on the expression of HIF- $1 \alpha$ and HO-1 after ischemia, the rats were randomly divided into two groups: Ischemia and reperfusion (I/R) and $\mathrm{EA}+\mathrm{I} / \mathrm{R}$. The animals were subjected to middle cerebral arterial occlusion (MCAO). The rats in the $\mathrm{EA}+\mathrm{I} / \mathrm{R}$ group were given 30 min of EA stimulation per day for 5 days. At $24 \mathrm{~h}$ after the last EA pretreatment and at 2, 6, 24, 48, and $72 \mathrm{~h}$ after reperfusion, RT-PCR and Western blot analysis were performed $(n=8)$. At $24 \mathrm{~h}$ after reperfusion, HIF- $1 \alpha / \mathrm{NeuN}$ double immunofluorescence analyses were performed in the Sham, I/R, and $\mathrm{EA}+\mathrm{I} / \mathrm{R}$ groups $(n=8)$.

\section{Experiment II}

To determine the effects of the HIF-1 $\alpha$ inhibitor $2 \mathrm{ME} 2$ on the neuroprotection associated with EA pretreatment, rats were randomly divided into five groups: Sham, I/R, $\mathrm{EA}+\mathrm{I} / \mathrm{R}, 2 \mathrm{ME} 2+\mathrm{I} / \mathrm{R}, \mathrm{EA}+2 \mathrm{ME} 2+\mathrm{I} / \mathrm{R} .2 \mathrm{ME} 2$ was administered by intraperitoneal (i.p.) injection at $30 \mathrm{~min}$ before MCAO in the $2 \mathrm{ME} 2+\mathrm{I} / \mathrm{R}$ and $\mathrm{EA}+2 \mathrm{ME} 2+\mathrm{I} / \mathrm{R}$ groups. At $72 \mathrm{~h}$ after reperfusion, neurobehavioral evaluation and infarct assessment were then performed $(n=8)$. At $24 \mathrm{~h}$ after reperfusion, TUNEL staining and both $\mathrm{Bcl} 2$ and Bax expressions were tested to measure neuronal apoptosis $(n=8)$.

\section{Experiment III}

To evaluate the interaction between the Notch signaling pathway and HIF-1 $\alpha$ levels induced by EA pretreatment, rats were randomly assigned to four groups $(n=8$ in each group): I/R, EA + I/R, EA + MW167 + I/R ( $\gamma$-secretase inhibitor) and EA + 2ME2 + I/R. MW167 was administered by intracerebroventricular (i.c.v.) injection $30 \mathrm{~min}$ before $\mathrm{MCAO}$ in the $\mathrm{EA}+\mathrm{MW} 167+\mathrm{I} / \mathrm{R}$ group, and 2ME2 was administered by i.p. injection $30 \mathrm{~min}$ before $\mathrm{MCAO}$ in the $\mathrm{EA}+2 \mathrm{ME} 2+\mathrm{I} / \mathrm{R}$ group. At $24 \mathrm{~h}$ after reperfusion, Western blot analysis and immunofluorescence were performed $(n=8)$.

\section{Electroacupuncture Pretreatment}

The EA pretreatment was performed as described previously (Wang et al. 2009). The "Baihui (GV 20)" acupoint was used, which, in rats, is located at the intersection of the sagittal midline and the line linking the two ears. The animals were anesthetized using $40 \mathrm{mg} / \mathrm{kg}$ sodium pentobarbital (SP) (i.p.), and then the acupoint "Baihui (GV 20) was stimulated at the intensity of $1 \mathrm{~mA}$ and frequency of $2 / 15 \mathrm{~Hz}$ for $30 \mathrm{~min}$ per day for five successive days using the G6805-2 EA Instrument (Model No. 227 033; Qingdao Xinsheng Ltd).

\section{Drug administration}

In experiment II, 2ME2 was dissolved in PBS with $10 \%$ dimethyl sulfoxide (DMSO) and administered by i.p. injection $30 \mathrm{~min}$ before $\mathrm{MCAO}$ in the $2 \mathrm{ME} 2+\mathrm{I} / \mathrm{R}$ and $\mathrm{EA}+2 \mathrm{ME} 2+\mathrm{I} / \mathrm{R}$ groups. The same volume of vehicle (10\% DMSO in PBS) was administered by i.p. injection 30 min before MCAO in Sham, I/R, and EA + I/R groups. The $2 \mathrm{ME} 2$ dose $(16 \mathrm{mg} / \mathrm{kg})$ was consistent with previously published in vivo doses (Zhou et al. 2008). In experiment III, MW167 was dissolved in PBS with $10 \%$ DMSO and used at a concentration of $1 \mathrm{mM}$ (Jurynczyk et al. 2005), which was administered by i.c.v. injection $30 \mathrm{~min}$ before MCAO in the EA + MW167 + I/R group. The same volume of vehicle(10\% DMSO in PBS) was administered by i.c.v. injection $30 \mathrm{~min}$ before $\mathrm{MCAO}$ in $\mathrm{I} / \mathrm{R}, \mathrm{EA}+\mathrm{I} / \mathrm{R}$, 
and $\mathrm{EA}+2 \mathrm{ME} 2+\mathrm{I} / \mathrm{R}$ groups. The i.c.v. injection was performed as described previously (Zhao et al. 2012). 2ME2 was dissolved in PBS with $10 \%$ dimethyl sulfoxide (DMSO) and administered by i.p. injection $30 \mathrm{~min}$ before $\mathrm{MCAO}$ in $\mathrm{EA}+2 \mathrm{ME} 2+\mathrm{I} / \mathrm{R}$ group. The same volume of vehicle (10\% DMSO in PBS) was administered by i.p. injection $30 \mathrm{~min}$ before $\mathrm{MCAO}$ in $\mathrm{I} / \mathrm{R}, \mathrm{EA}+\mathrm{I} / \mathrm{R}$, and $\mathrm{EA}+\mathrm{MW} 167+\mathrm{I} / \mathrm{R}$ groups. The stereotactic coordinates were as follows: $\mathrm{A},-1.0 ; \mathrm{R}, 1.5$; and $\mathrm{H}, 3.8$ (coordinates corresponding to the right lateral ventricle) (Paxinos et al. 1980).

\section{Transient Focal Cerebral Ischemia}

Animal Care: The animals were provided by the Experimental Animal Center of the Fourth Military Medical University (Xi'an, China). Male Sprague-Dawley (SD) rats weighing 260-300 g were housed in a specific pathogenfree environment with free access to sterile laboratory pellets and water. The experimental protocol was approved by the Ethics Committee for Animal Experimentation of the Fourth Military Medical University and was conducted according to the Guidelines for Animal Experimentation of the Fourth Military Medical University.

Focal cerebral ischemia was induced by a transient right middle cerebral artery occlusion (MCAO) in rats as described previously (Wang et al. 2012b). Animals were anesthetized by intraperitoneal injection of $40 \mathrm{mg} / \mathrm{kg} \mathrm{SP}$ for all surgical procedures. The regional cerebral blood flow (rCBF) was monitored using a disposable microtip fiber optic probe (diameter, $0.5 \mathrm{~mm}$ ) connected through a master probe to a computerized laser Doppler main unit (PeriFlux 5000, Perimed AB, Sweden). Rats retaining $>20 \%$ of baseline perfusion during ischemia were excluded. Reperfusion was accomplished by withdrawing the suture after $120 \mathrm{~min}$ of ischemia, followed by suturing of the surgical wounds. The temporalis muscle temperature was monitored and maintained at $37.0-37.5^{\circ} \mathrm{C}$ by surface heating or cooling during surgery until the rats recovered from anesthesia.

\section{Real-Time PCR}

The ischemic penumbras of the cerebral cortex were harvested as described previously (Wei et al. 2014). PCR was performed under the following thermal cycling conditions: one cycle at $94{ }^{\circ} \mathrm{C}$ for $5 \mathrm{~min} ; 25$ cycles at $94{ }^{\circ} \mathrm{C}$ for $30 \mathrm{~s}$, $55^{\circ} \mathrm{C}$ for $60 \mathrm{~s}$, and $72{ }^{\circ} \mathrm{C}$ for $30 \mathrm{~s}$ and one cycle at $72{ }^{\circ} \mathrm{C}$ for $10 \mathrm{~min}$. Melt-curve analysis was used to identify different reaction products, including nonspecific products. The following primers for RT-PCR were designed by the TaKaRa corporation: HIF-1 $\alpha$ (Fwd: CCA GAT TCA AGA TCA GCC AGC A, Rev: GCT GTC CAC ATC AAA GCA
GTA CTC A), HO-1 (Fwd: AGG TGC ACA TCC GTG CAG AG, Rev: CTT CCA GGG CCG TAT AGA TAT GGT A), and GAPDH (Fwd: GGC ACA GTC AAG GCT GAG AAT G, Rev: ATG GTG GTG AAG ACG CCA GTA). Each sample was tested in triplicate. Samples were obtained from 3 independent experiments and analyzed for relative gene expression data using the $2^{-\Delta \Delta \mathrm{CT}}$ method.

\section{Western Blot}

At $24 \mathrm{~h}$ after the last EA pretreatment and at 2, 6, 24, 48, and $72 \mathrm{~h}$ after reperfusion, HIF-1 $\alpha$ expression in the ischemic penumbra of rats in Experiment I was evaluated. At $24 \mathrm{~h}$ after reperfusion, the $\mathrm{Bcl} 2$ and Bax expression in the ischemic penumbra of rats in Experiment II was evaluated. At $24 \mathrm{~h}$ after reperfusion, NICD and HIF-1 $\alpha$ expression was measured in the rats in Experiment III as described previously (Deng et al. 2013, 2014). The following primary antibodies were used: mouse anti-HIF-1 $\alpha$ (1:400, Novus Biologicals), mouse anti-Bcl2 (1:200, Aobo), mouse antiBax (1:200, Aobo), rabbit anti-Notch1 NICD (1:400; Abcam), and mouse anti-beta-actin antibody (1:1000, Abcam). Secondary horseradish peroxidase (HRP)-conjugated goat-anti-rabbit antibody or goat-anti-mouse antibody (Pierce Biotechnology Inc; 1:5000 dilution) was used. Changes in relative protein expression were represented as the ratio of the integrated optical density of the protein bands to that of $\beta$-actin. Quantitative analysis of the protein bands was performed using an Image-Quant 5.0 GE Healthcare Densitometer (GE Healthcare, Sunnyvale, CA). The experiments were performed independently in triplicate.

\section{Double Immunofluorescence Staining}

HIF-1 $\alpha / \mathrm{NeuN}$ double immunofluorescence analyses were performed in the Sham, CON, and EA groups. Monoclonal mouse anti-HIF-1 $\alpha$ (1:200, Novus Biologicals) and polyclonal rabbit anti-NeuN (1:1000, Millipore) were used. HIF-1 $\alpha /$ Notch1 NICD double immunofluorescence analyses of the CON and EA groups were then performed. The secondary antibody used was FITC-labeled goat antimouse IgG (1:2000; Molecular Probes, USA) and Alexa Fluor 594-conjugated anti-rabbit IgG (1:1 000; Molecular Probes, USA). Finally, sections were observed, and images were captured using an Olympus BX-60 fluorescence microscope (Olympus Corporation, Shinjuku, Tokyo, Japan) with software named QCapture Pro.

\section{TUNEL Staining}

At $24 \mathrm{~h}$ after ischemia/reperfusion, TUNEL staining of Sham, I/R, EA + I/R, $2 \mathrm{ME} 2+\mathrm{I} / \mathrm{R}, \mathrm{EA}+2 \mathrm{ME} 2+\mathrm{I} / \mathrm{R}$ 
groups from Experiment II was performed using an In Situ Cell Death Detection Kit (Roche Diagnostics, Mannheim, Germany) as described previously(Wang et al. 2013) and according to the manufacturer's instructions. Images were viewed using an Olympus BX-60 fluorescence microscope. Integrated optical density/area of positive TUNEL staining in each group was measured by 2 blinded investigators using Image-Pro plus 5.1 software (Media Cybernetics, Inc., Bethesda, MD, USA).

\section{Neurobehavioral Evaluation and Infarct Volume Assessment}

At $72 \mathrm{~h}$ after reperfusion, a neurological assessment of the rats in Sham, I/R, EA + I/R, 2ME2 + I/R, EA + 2ME2 + $\mathrm{I} / \mathrm{R}$ groups was performed by a blinded observer using the 18-point scoring system reported by Garcia et al. (1995). The system consisted of the following six tests: (1) spontaneous activity, (2) symmetry in the movement of four limbs, (3) forepaw outstretching, (4) climbing, (5) body proprioception, and (6) response to vibrissae touch. The score given to each rat at the completion of the evaluation was the summation of all six individual test scores. Minimum neurologic score was 3; maximum was 18 .

After neurological evaluation, rats were decapitated, and 2-mm thick coronal sections throughout the brain were stained with $2 \%$ 2,3,5-triphenyltetrazolium chloride (TTC) to evaluate the infarct volume as described previously(Zhou et al. 2013). The areas of injured (white) and uninjured (red) were measured using an image analysis system (Adobe Photoshop 8.0, Adobe Systems Incorporated, San Jose, CA) for each slice. Infarct volume was calculated by Swanson and Sharp's method to correct for edema: $100 \times$ (contralateral hemisphere volume-nonlesioned ipsilateral hemisphere volume)/contralateral hemisphere volume (Swanson and Sharp 1994).

\section{Statistical Analysis}

SPSS 11.0 for Windows (SPSS Inc., Chicago, IL) was used. The HIF- $1 \alpha$ and HO-1 mRNA levels, HIF-1 $\alpha, \mathrm{Bcl} 2, \mathrm{Bax}$ and NICD levels and infarct volumes were analyzed using one-way analysis of variance, and differences between the groups were detected using the post hoc Student-NewmanKeuls test. Each neurological deficit score was expressed as a median (range) and analyzed using the Kruskal-Wallis test followed by Dunn's post hoc test. Other values were reported as the mean \pm standard error of the mean (SEM) and analyzed among groups using one-way analysis of variance. When indicated by a significant $F$ ratio, post hoc testing was performed using Scheffe's test. Values of $p<0.05$ were considered statistically significant.

\section{Results}

\section{EA Pretreatment Significantly Enhanced HIF-1 $\alpha$ and HO-1 Expression in the Ischemic Penumbra at the Early Stage of Reperfusion}

Expression of the HIF-1 $\alpha$ and HO- 1 genes in the ischemic penumbra was examined at the early stage of reperfusion. HO- 1 is one of the downstream genes regulated by HIF- $1 \alpha$. The HIF-1 $\alpha$ and HO-1 mRNA expression levels were significantly higher in EA $+\mathrm{I} / \mathrm{R}$ group than in I/R group at 2,6 , and $24 \mathrm{~h}$ after reperfusion $(p<0.05)$. However, the expression levels of HIF-1 $\alpha$ and HO-1 mRNA were significantly lower in EA $+\mathrm{I} / \mathrm{R}$ group than in $\mathrm{I} / \mathrm{R}$ group at 48 and $72 \mathrm{~h}$ after reperfusion $(p<0.05)($ Fig. $1 \mathrm{~b} / \mathrm{c})$.

Semi-quantitative Western blot analysis indicated that HIF-1 $\alpha$ was significantly elevated at $2 \mathrm{~h}$ after reperfusion in $\mathrm{EA}+\mathrm{I} / \mathrm{R}$ group ( $p<0.05$ vs. before $\mathrm{I} / \mathrm{R}$ ) and reached the maximum level at $48 \mathrm{~h}$ after reperfusion. Furthermore, the level of HIF-1 $\alpha$ in the $\mathrm{EA}+\mathrm{I} / \mathrm{R}$ group was significantly higher than in I/R group $(p<0.05)$ at $2,6,24$, or 48 h (Fig. 1d, e).

We also evaluated whether neurons in the ischemic penumbra region expressed HIF-1 $\alpha$. The results indicated that there were more HIF-1 $\alpha$ - and NeuN-positive cells in $\mathrm{EA}+\mathrm{I} / \mathrm{R}$ group than in I/R group $24 \mathrm{~h}$ after reperfusion. Furthermore, the number of HIF- $1 \alpha / \mathrm{NeuN}$ double-labeled neurons in $\mathrm{EA}+\mathrm{I} / \mathrm{R}$ group was significantly higher than in I/R group $24 \mathrm{~h}$ after reperfusion (Fig. 2). These results suggested that EA pretreatment increased the expression of HIF- $1 \alpha$ and HO-1 in the ischemic penumbra at the early stage of reperfusion.

\section{HIF-1 $\alpha$ Inhibitor 2ME2 Attenuated the Neuroprotective Effect of EA Pretreatment}

Neurological function was assessed using Garcia scores. At $72 \mathrm{~h}$ after reperfusion, rats receiving EA pretreatment showed significantly higher neurological function scores than those in I/R group $(p<0.05)$. However, the neurological function scores in the group given EA pretreatment with 2ME2 were significantly lower than in the group pretreated with EA alone (Fig. 3a).

At $72 \mathrm{~h}$ after reperfusion, the brain infarct volumes in $\mathrm{EA}+\mathrm{I} / \mathrm{R}$ group were significantly smaller than those in $\mathrm{I} / \mathrm{R}$ group $(p<0.05)$. Interestingly, the infarct volumes in $\mathrm{EA}+2 \mathrm{ME} 2+\mathrm{I} / \mathrm{R}$ group were significantly larger than those in $\mathrm{EA}+\mathrm{I} / \mathrm{R}$ group $(p<0.05)$. There was no difference between the I/R group and the group subjected to only $2 \mathrm{ME} 2$ (Fig. 3b, c). These results suggested that the neuroprotective effect induced by EA pretreatment can be alleviated by $2 \mathrm{ME} 2$ intervention. 
A

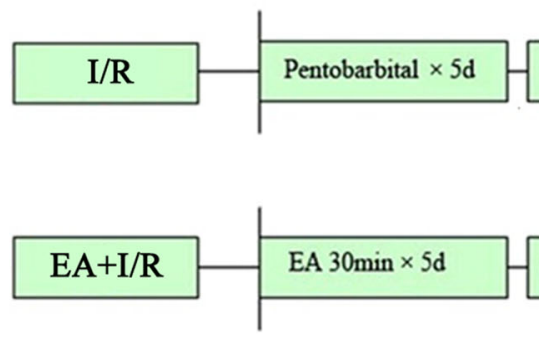

B

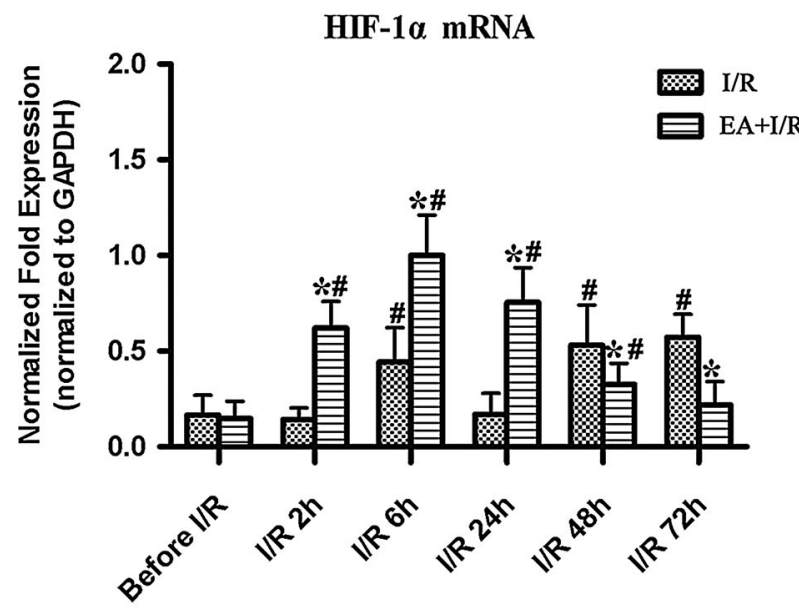

D

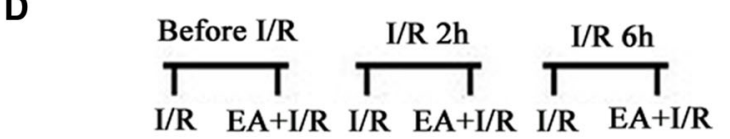

HIF-1 $\alpha$

$\beta$-Actin

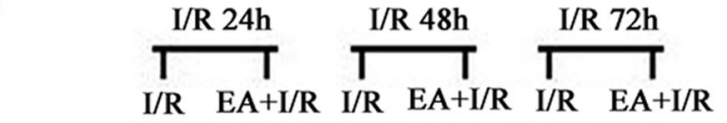

HIF- $1 \alpha$

$\beta$-Actin

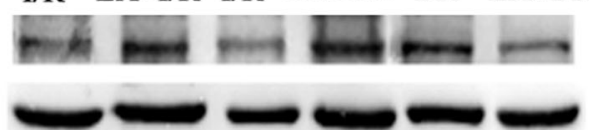

Fig. 1 The expression of HIF-1 $\alpha$ and HO-1 in the ischemic penumbra at $24 \mathrm{~h}$ after the final EA pretreatment and at 2, 6, 24, 48 , and $72 \mathrm{~h}$ after ischemia/reperfusion $(n=8)$. a Experimental protocol. Red arrows indicate the time points at which the RT-PCR and Western blot analyses of HIF- $1 \alpha$ or the RT-PCR analyses of HO1 were performed. b RT-PCR analysis of the HIF- $1 \alpha$ mRNA levels in

To assess the effect of 2ME2 on neuronal apoptosis, TUNEL staining on ischemic brain sections at $24 \mathrm{~h}$ after reperfusion was performed. There were more TUNELpositive cells in the ischemic penumbras from the I/R and $\mathrm{EA}+2 \mathrm{ME} 2+\mathrm{I} / \mathrm{R}$ groups than from the $\mathrm{EA}+\mathrm{I} / \mathrm{R}$ group $(p<0.05) 24 \mathrm{~h}$ after reperfusion (Fig. 4b, c, e). These

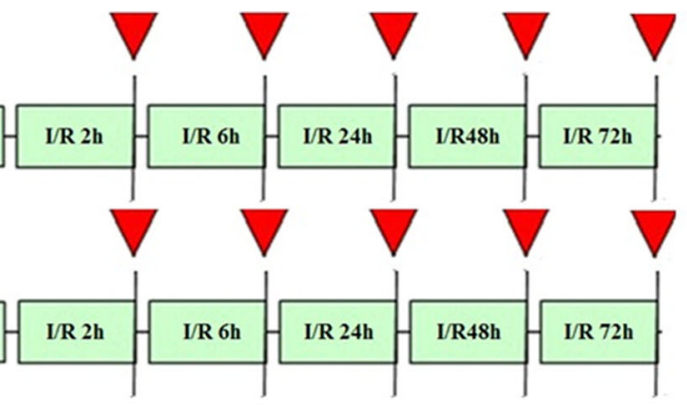

C
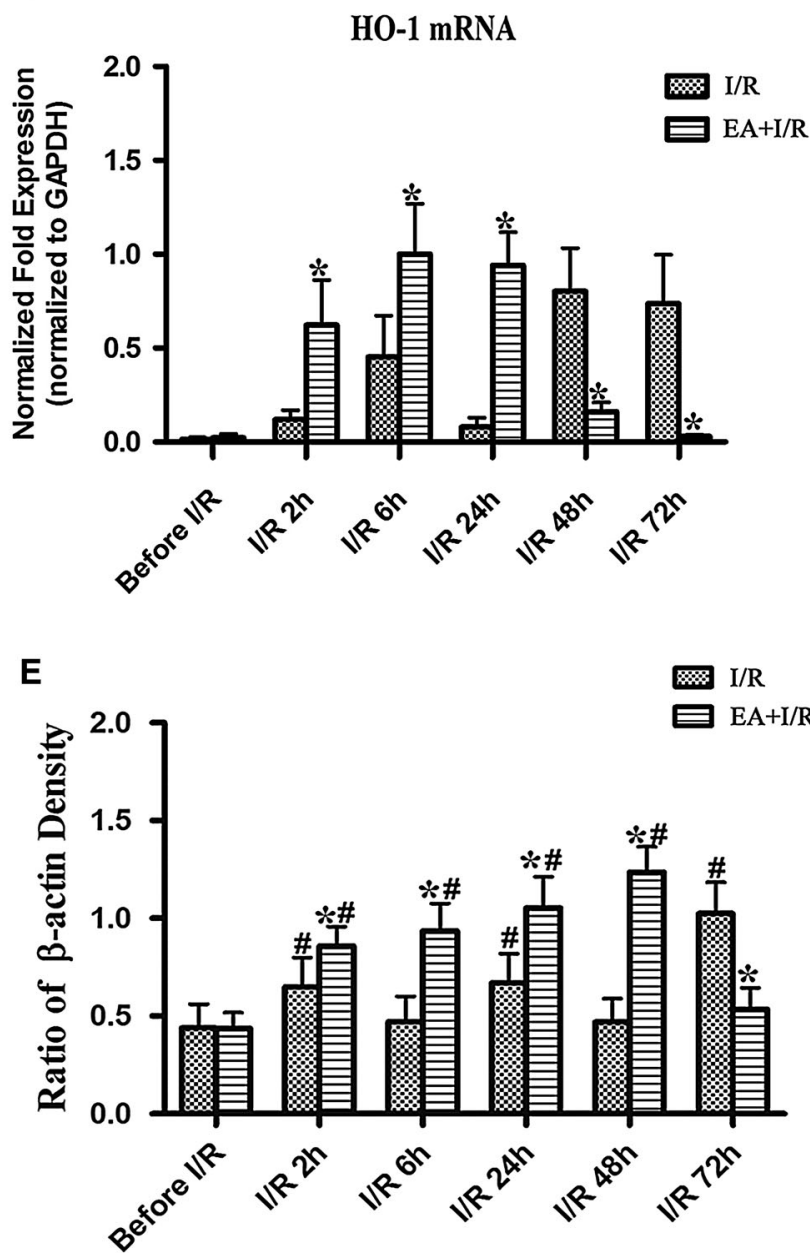

the ischemic penumbra. c RT-PCR analysis of the HO-1 mRNA levels in the ischemic penumbra. d Representative Western blot bands showing HIF-1 $\alpha$ expression in rats between I/R and EA + I/R groups. e Bar graph showing quantification of the Western blot analysis comparing the HIF- $1 \alpha$ protein with $\beta$-actin $(* p<0.05$ vs. I/R; $\# p<0.05$ vs. before I/R)

results indicated that the EA pretreatment-induced reduction of neuronal apoptosis was attenuated by $2 \mathrm{ME} 2$.

We also assayed the expressions of the Bax and Bcl-2 proteins $24 \mathrm{~h}$ after reperfusion. As shown in Fig. 3g-i, rats only subjected to the EA pretreatment showed markedly up-regulated Bcl-2 levels ( $p<0.05$ vs. I/R) in the ischemic 
Fig. 2 Immunofluorescence staining detected the expression of HIF- $1 \alpha$ in neurons in the ischemic penumbra at $24 \mathrm{~h}$ after reperfusion. a Representative double immunofluorescence staining (yellow) of HIF-1 $\alpha$ positive cells (green) and NeuN-positive cells $(\mathrm{red})$ in brain sections was displayed. Scale bars $100 \mu \mathrm{m}$. b Statistical analysis of the HIF- $1 \alpha$-positive cell numbers in the observed area. c Statistical analysis of the NenN-positive cell numbers in the observed area. d Statistical analysis of the HIF- $1 \alpha / \mathrm{NenN}$ double labeling cell numbers in the observed area $(* p<0.05 \mathrm{vs}$. Sham; $\# p<0.05$ vs. I/R)
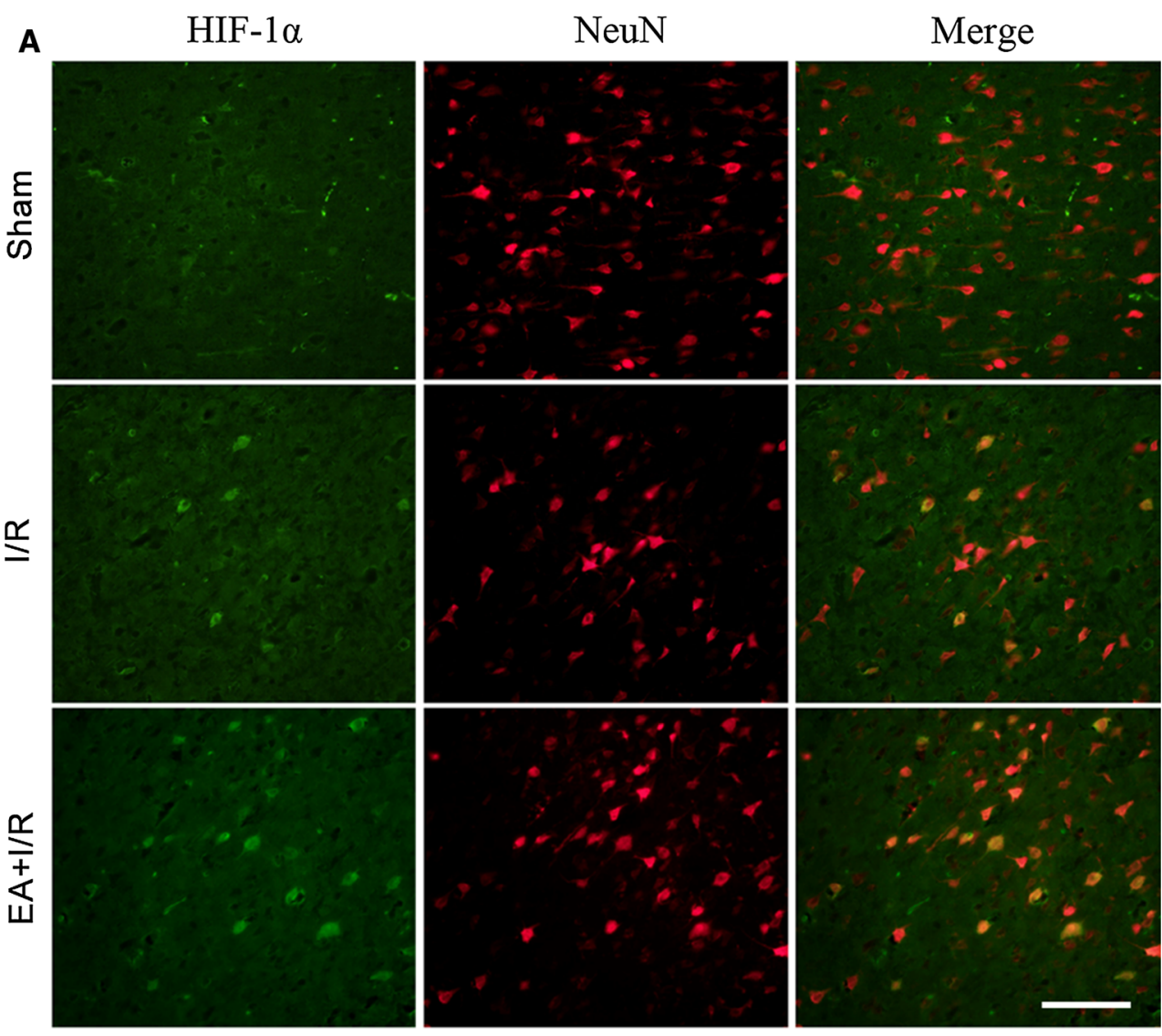

B

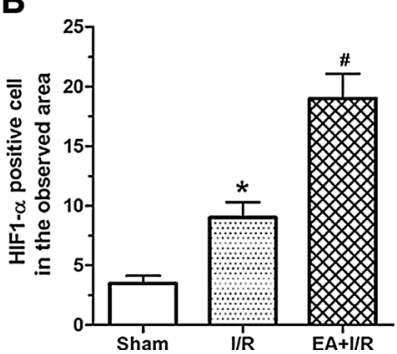

C

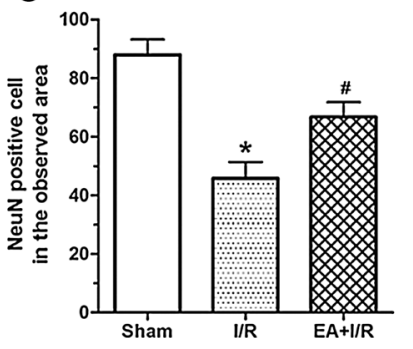

D

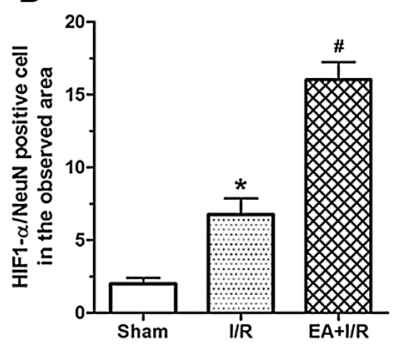

penumbra $24 \mathrm{~h}$ after reperfusion, whereas the Bcl-2 protein levels in the ischemic penumbra in EA $+2 \mathrm{ME} 2+\mathrm{I} / \mathrm{R}$ group were significantly lower than those in $\mathrm{EA}+\mathrm{I} / \mathrm{R}$ group $(p<0.05)($ Fig. $4 \mathrm{~g} / \mathrm{h} / \mathrm{i})$. Meanwhile, the up-regulation of Bax in the ischemic penumbra was markedly reduced by the EA pretreatment ( $p<0.05$ vs. I/R).

\section{Interaction Between the Notch Signaling Pathway and HIF-1 $\alpha$}

As shown in Fig. 5b-e, there were significantly more HIF$1 \alpha$ - or NICD-positive cells in the ischemic penumbra in $\mathrm{EA}+\mathrm{I} / \mathrm{R}$ group than in $\mathrm{I} / \mathrm{R}$ group $(p<0.05) 24 \mathrm{~h}$ after reperfusion. Furthermore, there were more HIF-1 $\alpha /$ NICD double-labeled cells in the ischemic penumbra in EA $+\mathrm{I} / \mathrm{R}$ group than in $\mathrm{I} / \mathrm{R}$ group $(\mathrm{p}<0.05) 24 \mathrm{~h}$ after reperfusion.
These results indicated that HIF-1 $\alpha$ and Notch1NICD were co-expressed in the ischemic penumbra.

To explore the relationship between the Notch signaling pathway and HIF- $1 \alpha$, we evaluated the expression of HIF- $1 \alpha$ and NICD after the Notch signal was inhibited. As shown in Fig. 5f, g, the amount of HIF- $1 \alpha$ and NICD in the ischemic penumbra of $\mathrm{EA}+\mathrm{I} / \mathrm{R}$ group was significantly higher than that in $\mathrm{I} / \mathrm{R}$ group $(p<0.05) 24 \mathrm{~h}$ after reperfusion. However, MW167, which can inhibit the activation of the Notch signaling pathway, decreased the expressions of both HIF- $1 \alpha$ and NICD $(p<0.05, \mathrm{EA}+\mathrm{MW} 167+\mathrm{I} / \mathrm{R}$ vs. EA + I/R). Furthermore, 2ME2 inhibited HIF-1 $\alpha$ expression $(p<0.05$, EA $+2 \mathrm{ME} 2+$ $\mathrm{I} / \mathrm{R}$ vs. EA $+\mathrm{I} / \mathrm{R}$ ) but had no effect on the expression of NICD $(p>0.05, \mathrm{EA}+2 \mathrm{ME} 2+\mathrm{I} / \mathrm{R}$ vs. EA $+\mathrm{I} / \mathrm{R})$. These results suggested that inhibition of the Notch signal suppressed the expression of HIF- $1 \alpha$ in the ischemic penumbra. 

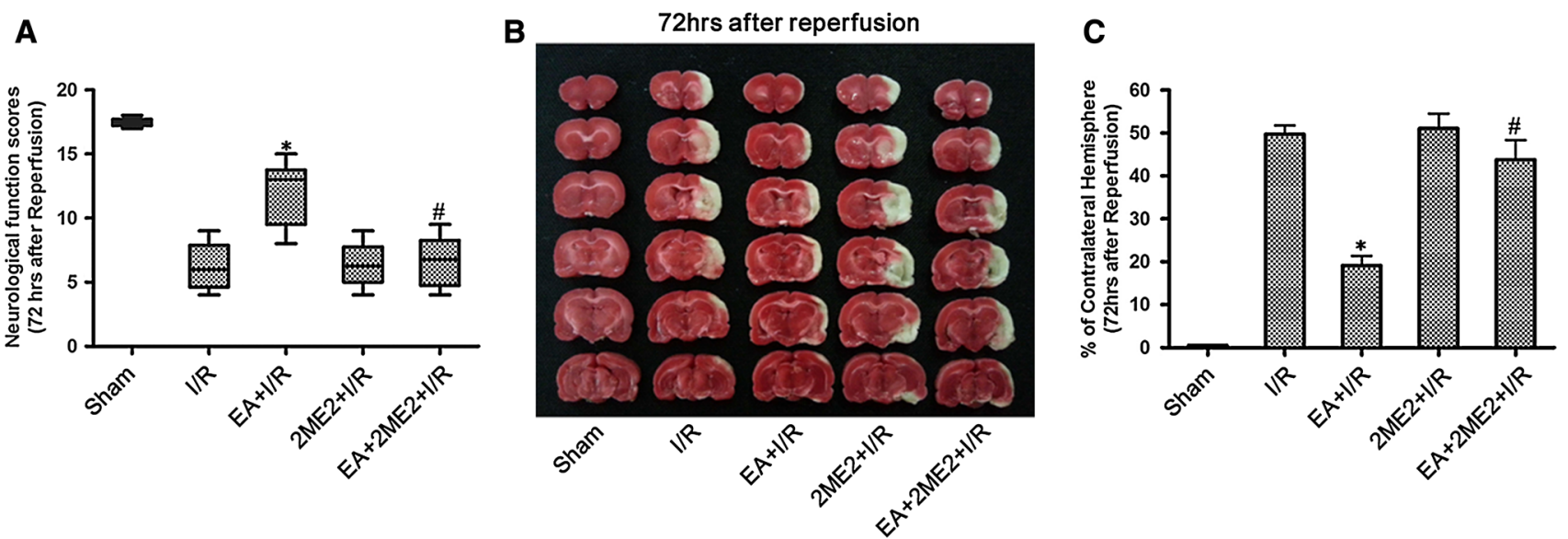

D
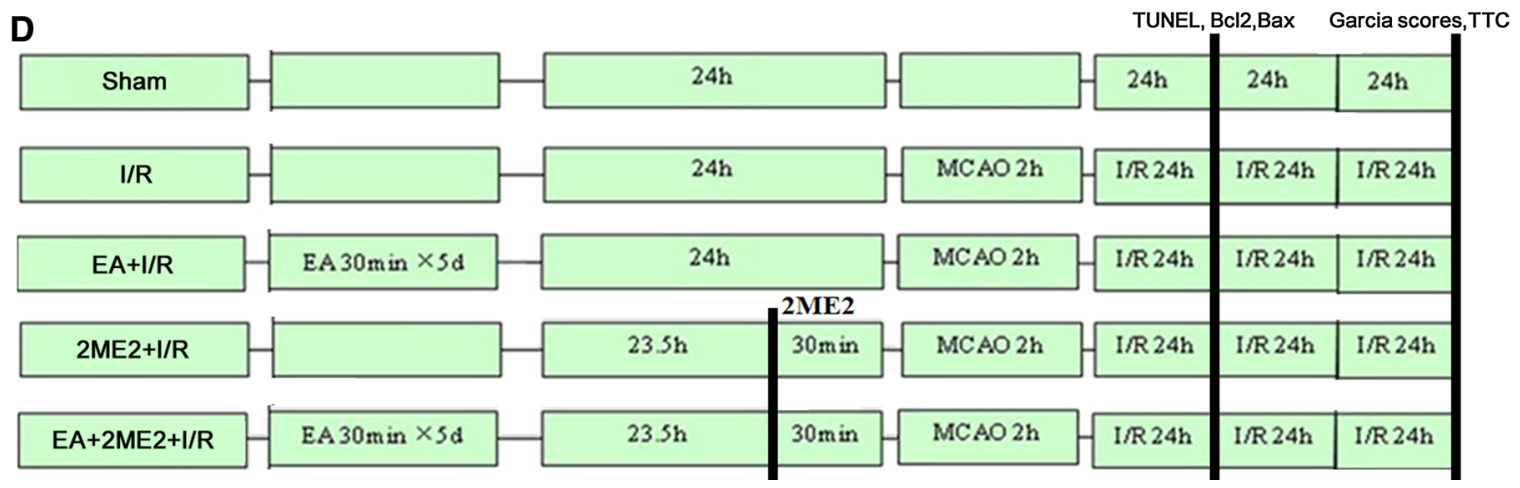

Fig. 3 Neurological scores and infarct volumes at $72 \mathrm{~h}$ after reperfusion in the rats. a Garcia scores were tested at $72 \mathrm{~h}$ after reperfusion. b Representative brain infarct size indicated by TTC staining at $72 \mathrm{~h}$ after reperfusion. c Statistical analysis of the infarct size in every group (\% of contralateral hemisphere) $(* p<0.05$ vs. I/R; \#p $<0.05$ vs. EA + I/R). d Experimental protocol, SD rats were randomly divided into five groups $(n=8$ each): Sham, I/R,

\section{Discussion}

In the present study, we found that EA pretreatment significantly increased HIF-1 $\alpha$ expression in the ischemic penumbra following reperfusion. In addition, immunofluorescence staining suggested that HIF-1 $\alpha$ immunoreactivity was colocalized with NeuN immunoreactivity, indicating that the effect of EA pretreatment on HIF-1 $\alpha$ expression may be neuron-specific. Baranova et al. reported that the neuron-specific inactivation of HIF-1 $\alpha$ increased brain injury in a mouse model of transient focal cerebral ischemia (Baranova et al. 2007). A research showed that sevoflurane postconditioning protected the brain from focal cerebral ischemic reperfusion injury through up-regulating mRNA and protein expression of HIF- $1 \alpha$ and its target gene, HO-1 (Ye et al. 2012). To address whether HIF-1 $\alpha$ played a neuroprotective role in EA pretreatment after reperfusion, we inhibited HIF-1 $\alpha$ expression using 2ME2, which selectively suppressed cellular HIF-1 $\alpha$ protein synthesis without affecting HIF-1 $\alpha$
$2 \mathrm{ME} 2+\mathrm{I} / \mathrm{R}, \mathrm{EA}+\mathrm{I} / \mathrm{R}$, and $\mathrm{EA}+2 \mathrm{ME} 2+\mathrm{I} / \mathrm{R}$ group. At $30 \mathrm{~min}$ before MCAO, the $2 \mathrm{ME} 2$ were administered. At $72 \mathrm{~h}$ after ischemia/ reperfusion, neurological function scores, and infarct volumes were assessed in every group. At $24 \mathrm{~h}$ after ischemia/reperfusion, TUNEL staining, the expression of $\mathrm{Bcl} 2$ and Bax was tested to evaluate neuronal apoptosis in every group

mRNA transcription or the stability of the HIF-1 $\alpha$ protein (Baranova et al. 2007). 2ME2 is a natural metabolite of estrogen that is known to inhibit HIF-1 $\alpha$ in a dose-dependent manner (Ricker et al. 2004). The dose (16 mg/kg) of 2ME2 can effectively inhibit the expression of HIF-1 $\alpha$ and its target gene, VEGF(Zhou et al. 2008; Zhu et al. 2014). The administration of 2ME2 attenuated the beneficial effects of EA on infarct volumes, neurological outcomes, and apoptosis. These results suggest that the up-regulation of HIF-1 $\alpha$ may contribute to the neuroprotective effects of EA pretreatment against focal ischemia. Certainly, 2ME2 has other features, such as anti-tumor, anti-angiogenesis, anticytotoxicity, and anti-proliferation. These features may have some effect on our research.

Apoptosis is one of the major causes of neuronal injury following cerebral ischemia, and the inhibition of apoptosis reduces ischemic injury. HIF-1 $\alpha$ is a transcription factor that plays a key role in regulating the adaptive response to hypoxia and is involved in regulating apoptosis under hypoxic conditions. Increasing HIF- $1 \alpha$ protein stability 

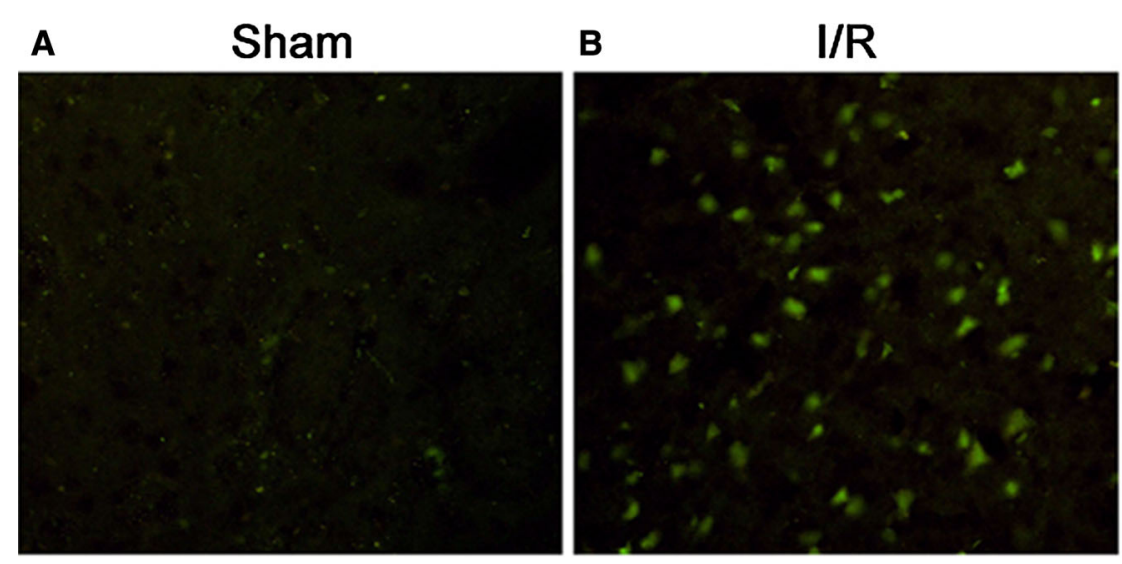

\section{C $\quad E A+I / R$}
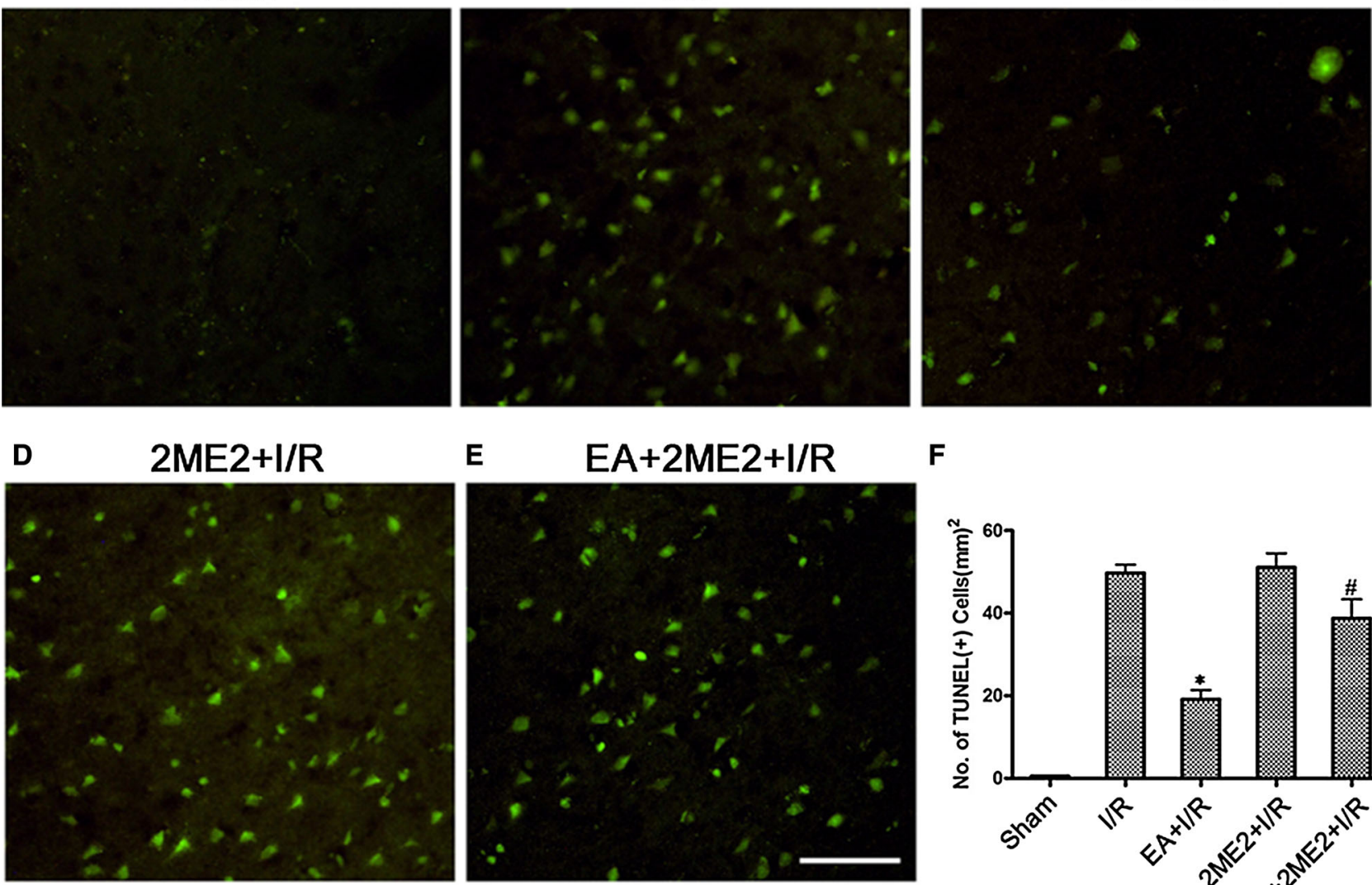

$\mathbf{F}$

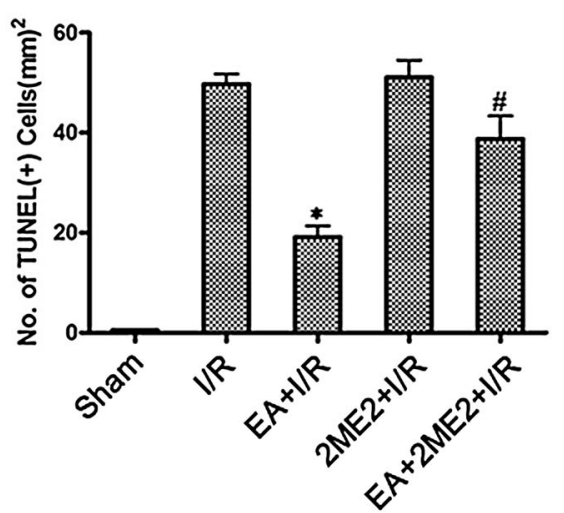

H

I
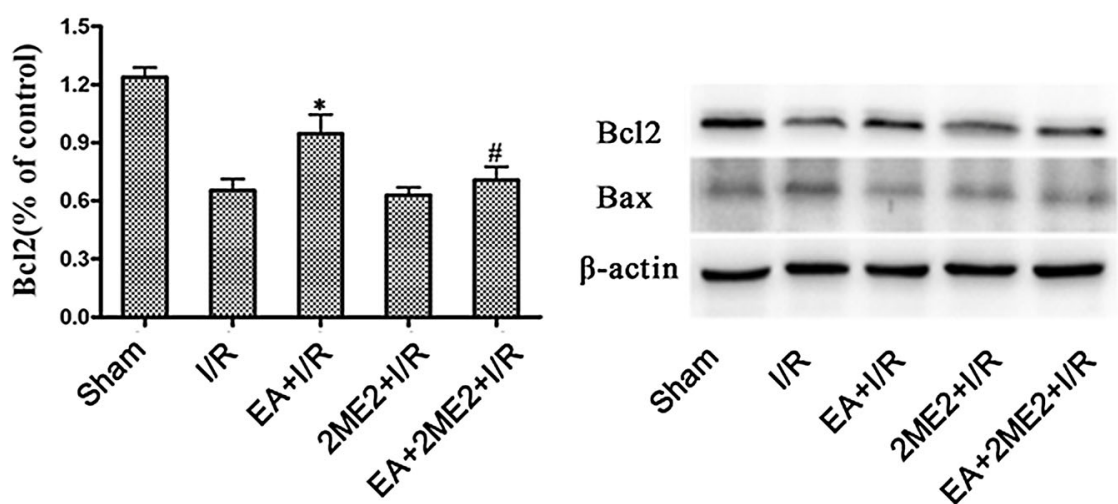

Fig. 4 The representative TUNEL staining and the expression of $\mathrm{Bcl}-2$ and Bax protein was tested at $24 \mathrm{~h}$ after reperfusion in every group. a-e The representative immunofluorescence staining of TUNEL-positive cells (green) in brain sections was displayed. Scale bars $100 \mu \mathrm{m}$. f Statistical analysis of the TUNEL-positive cells numbers. h Representative Western blot bands of Bcl-2 and Bax expressions at $24 \mathrm{~h}$ after reperfusion. $\mathbf{g} / \mathbf{i}$ Statistical analysis comparing both $\mathrm{Bcl}-2$ and $\mathrm{Bax}$ protein expression with $\beta$-actin $\left({ }^{*} p<0.05\right.$ vs. $\mathrm{I} / \mathrm{R} ; \# p<0.05$ vs. $\mathrm{EA}+\mathrm{I} / \mathrm{R})$

neuronal apoptosis. Yang et al. reported that Panaxynol protected cortical neurons from ischemia-like injury by upregulation of HIF-1 $\alpha$ expression and inhibition of apoptotic cascade (Yang et al. 2010). However, how HIF-1 $\alpha$ exerts its neuroprotective effects after EA administered remains investigation. 


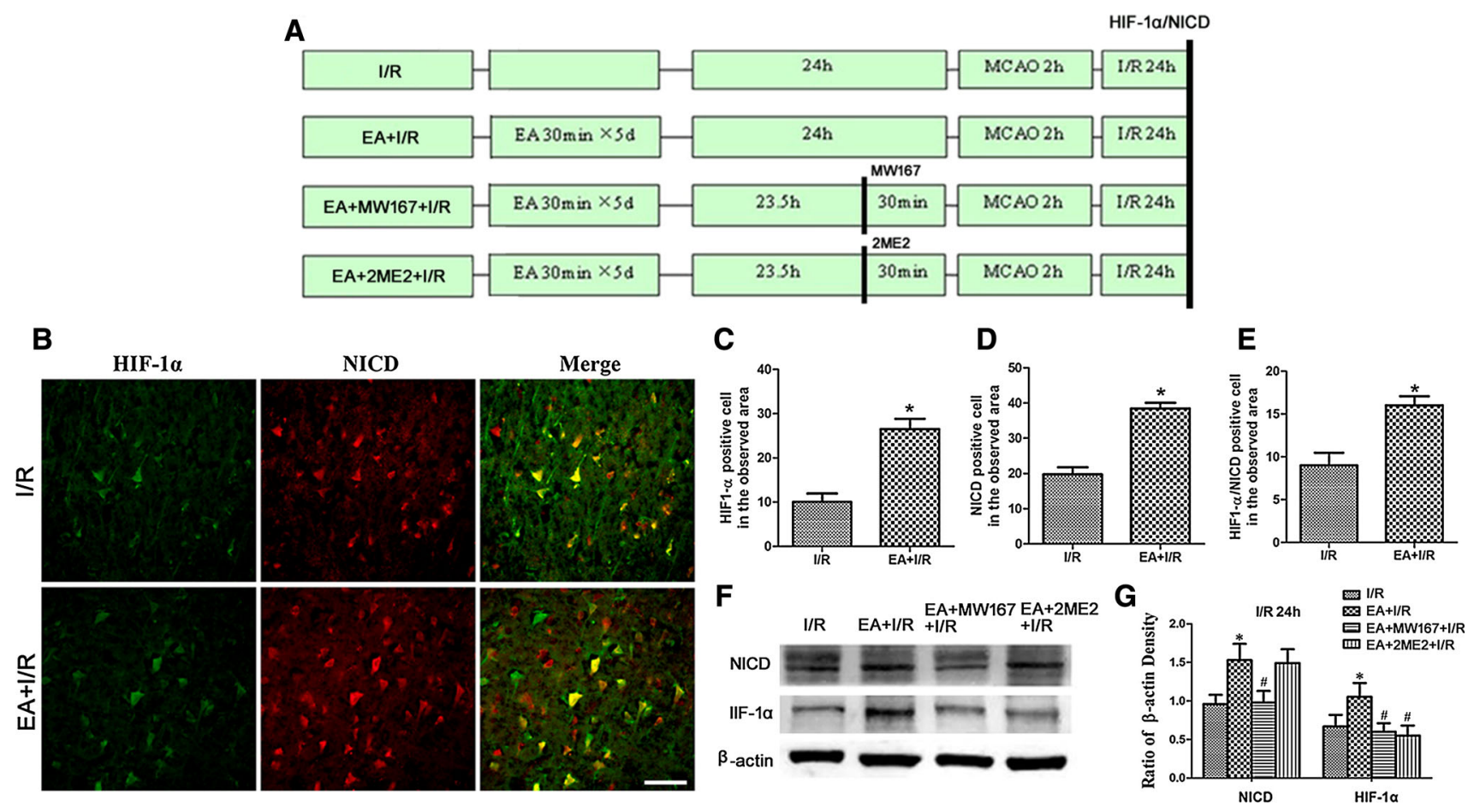

Fig. 5 Representative double immunofluorescence staining and Western blot analyzing the expression of the HIF- $1 \alpha$ and NICD at $24 \mathrm{~h}$ after reperfusion. a Experimental protocol. b Representative double immunofluorescence staining of HIF-1 $\alpha$-positive cells (green) and NICD-positive cells (red). Scale bars $50 \mu \mathrm{m}$. c Statistical analysis of the HIF-1 $\alpha$-positive cell numbers in the observed area. d Statistical analysis of the NICD-positive cell numbers in the observed area.

e Statistical analysis of the HIF-1 $\alpha /$ NICD double labeling cell numbers in the observed area $(* p<0.05$ vs. I/R). f Representative Western blot bands of HIF- $1 \alpha$ and Notch1 NICD expression in rats from the I/R, EA + I/R, EA + MW167 + I/R, and EA + 2ME2 + $\mathrm{I} / \mathrm{R}$ groups at $24 \mathrm{~h}$ after reperfusion. $\mathrm{g}$ Statistical analysis comparing the expressions of the HIF- $1 \alpha$ and NICD proteins with the expression of $\beta$-actin $(* p<0.05$ vs. I/R; $\# p<0.05$ vs. $\mathrm{EA}+\mathrm{I} / \mathrm{R})$

It is still controversial whether the activated canonical Notch signaling is beneficial to the ischemic cerebral tissues. Many researchers have found that the activation of Notch signaling was involved in the ischemic tolerance induced by inhalation anesthetics preconditioning (Zhang et al. 2014; Yang et al. 2012). However, other reports was consistent with the opinion that Notch signaling can induce neuronal cell death (Arumugam et al. 2011). The discrepancies might be explained by the injury model, the severity of injury and the timepoint of notch inhibition. In our past work, we found that EA pretreatment-induced tolerance against focal cerebral ischemia through activation of the canonical Notch signaling pathway (Zhao et al. 2012). Notch signaling and the cellular hypoxic response have been shown to be functionally integrated (Gustafsson et al. 2005; Kamarehei and Yazdanparast 2014). Zheng et al. reported that FIH-1 played a role in mediating hypoxia-potentiated Notch signaling, suggesting that Notch ICD could sequester FIH-1 from HIF$1 \alpha$, thus decreasing HIF- $1 \alpha$ function (Zheng et al. 2008). Lee $\mathrm{JH}$ et al. found that the transcription of HIF- $1 \alpha$ gene was upregulated by the induction of Notch signaling under hypoxic conditions (Lee et al. 2009). Our results also indicated the inhibition of the Notch signal suppressed HIF-1 $\alpha$ expression

in the ischemic penumbra, whereas HIF- $1 \alpha$ inhibition had no effect on NICD expression. These results suggest that the upregulation of HIF- $1 \alpha$ during the EA pretreatment is mediated by the activation of the Notch pathway. However, our results do not agree with a recent report by Cheng et al. showing that the NICD and HIF- $1 \alpha$ collaborated to engage pro-inflammatory and apoptotic signaling pathways in stroke (Cheng et al. 2014). The discrepancies might be explained by the different levels of HIF- $1 \alpha$ expression in the ischemic penumbra after focal cerebral ischemia, because HIF coordinated both cell survival and death mechanisms. The research of Cheng focused on the expression and function of NICD and HIF-1 $\alpha$ in ischemic stroke, while our focus was the benefit of up-regulation of NICD and HIF-1 $\alpha$ expression induced by EA pretreatment in the focal cerebral ischemic injury. Besides, the time of MCAO in Cheng' research was much less than that in our research.

In summary, the current investigation indicates that EA pretreatment affords strong protection against transient cerebral ischemic injury and increases the protein expression and activation of HIF- $1 \alpha$ in rats. The beneficial effects of EA pretreatment may be mediated by the activation of the Notch signaling pathway. 
Acknowledgments The authors thank Prof. Xude Sun for surgical assistance of MCAO model and guidance for writing. This work was supported by the National Natural Science Foundation of China (Grant Nos. 81471265, 81271290, 81271343 and 30772073), in part by the Natural Science Foundation of Shaanxi Province (Grant Nos. 2012KTCG01-02 and 2012FHA01), in part by the Science and Technology Project of Fujian Province (Grant No. 2012Y0054) and in part by the Fourth Military Medical University Foundation (Grant No. 2013-D03). The funders had no role in the study design, data collection and analysis, decision to publish or preparation of the manuscript.

Conflict of interest The authors declare no conflict of interest.

Open Access This article is distributed under the terms of the Creative Commons Attribution 4.0 International License (http:// creativecommons.org/licenses/by/4.0/), which permits unrestricted use, distribution, and reproduction in any medium, provided you give appropriate credit to the original author(s) and the source, provide a link to the Creative Commons license, and indicate if changes were made.

\section{References}

Arumugam TV, Cheng YL, Choi Y, Choi YH, Yang S, Yun YK et al (2011) Evidence that gamma-secretase-mediated Notch signaling induces neuronal cell death via the nuclear factor-kappaBBcl-2-interacting mediator of cell death pathway in ischemic stroke. Mol Pharmacol 1:23-31. doi:10.1124/mol.111.071076

Baranova O, Miranda LF, Pichiule P, Dragatsis I, Johnson RS, Chavez JC (2007) Neuron-specific inactivation of the hypoxia inducible factor 1 alpha increases brain injury in a mouse model of transient focal cerebral ischemia. J Neurosci 23:6320-6332. doi:10.1523/JNEUROSCI.0449-07.2007

Cheng YL, Park JS, Manzanero S, Choi Y, Baik SH, Okun E et al (2014) Evidence that collaboration between HIF-1alpha and Notch-1 promotes neuronal cell death in ischemic stroke. Neurobiol Dis. doi:10.1016/j.nbd.2013.10.009

Deng B, Gou X, Chen H, Li L, Zhong H, Xu H et al (2013) Targeted delivery of neurogenin-2 protein in the treatment for cerebral ischemia-reperfusion injury. Biomaterials 34:8786-8797. doi:10. 1016/j.biomaterials.2013.07.076

Deng B, Xu H, Zhang J, Wang J, Han LC, Li LY et al (2014) Neuroprotective effects of sevoflurane against electromagnetic pulse-induced brain injury through inhibition of neuronal oxidative stress and apoptosis. PLoS ONE 3:e91019. doi:10. 1371/journal.pone.0091019

Doeppner TR, Mlynarczuk-Bialy I, Kuckelkorn U, Kaltwasser B, Herz J, Hasan MR et al (2012) The novel proteasome inhibitor BSc2118 protects against cerebral ischaemia through HIF1A accumulation and enhanced angioneurogenesis. Brain 11:3282-3297. doi:10.1093/brain/aws269

Garcia JH, Wagner S, Liu KF, Hu XJ (1995) Neurological deficit and extent of neuronal necrosis attributable to middle cerebral artery occlusion in rats. Statistical validation. Stroke. 4(627-634):635

Go AS, Mozaffarian D, Roger VL, Benjamin EJ, Berry JD, Borden WB et al (2013) Heart disease and stroke statistics-2013 update: a report from the American Heart Association. Circulation 1:e6e245. doi:10.1161/CIR.0b013e31828124ad

Gustafsson MV, Zheng X, Pereira T, Gradin K, Jin S, Lundkvist J et al (2005) Hypoxia requires notch signaling to maintain the undifferentiated cell state. Dev Cell 5:617-628. doi:10.1016/j. devcel.2005.09.010
Jung JE, Lee HG, Cho IH, Chung DH, Yoon SH, Yang YM et al (2005) STAT3 is a potential modulator of HIF-1-mediated VEGF expression in human renal carcinoma cells. Faseb J 10:1296-1298. doi:10.1096/fj.04-3099fje

Jurynczyk M, Jurewicz A, Bielecki B, Raine CS, Selmaj K (2005) Inhibition of Notch signaling enhances tissue repair in an animal model of multiple sclerosis. J Neuroimmunol 1-2:3-10. doi:10. 1016/j.jneuroim.2005.10.013

Kamakura S, Oishi K, Yoshimatsu T, Nakafuku M, Masuyama N, Gotoh Y (2004) Hes binding to STAT3 mediates crosstalk between Notch and JAK-STAT signalling. Nat Cell Biol 6:547-554. doi:10.1038/ncb1138

Kamarehei M, Yazdanparast R (2014) Modulation of notch signaling pathway to prevent $\mathrm{H} 2 \mathrm{O} 2 /$ menadione-induced SK-N-MC cells death by EUK134. Cell Mol Neurobiol 7:1037-1045. doi:10. 1007/s10571-014-0079-0

Kunze R, Zhou W, Veltkamp R, Wielockx B, Breier G, Marti HH (2012) Neuron-specific prolyl-4-hydroxylase domain 2 knockout reduces brain injury after transient cerebral ischemia. Stroke 10:2748-2756. doi:10.1161/STROKEAHA.112.669598

Lee JH, Suk J, Park J, Kim SB, Kwak SS, Kim JW et al (2009) Notch signal activates hypoxia pathway through HES1-dependent SRC/ signal transducers and activators of transcription 3 pathway. Mol Cancer Res 10:1663-1671. doi:10.1158/1541-7786.MCR-09-0191

Lopez-Hernandez B, Posadas I, Podlesniy P, Abad MA, Trullas R, Cena V (2012) HIF-1alpha is neuroprotective during the early phases of mild hypoxia in rat cortical neurons. Exp Neurol 1:543-554. doi:10.1016/j.expneurol.2011.11.040

Paxinos G, Watson CR, Emson PC (1980) AChE-stained horizontal sections of the rat brain in stereotaxic coordinates. J Neurosci Methods 2:129-149

Ricker JL, Chen Z, Yang XP, Pribluda VS, Swartz GM, Van Waes C (2004) 2-methoxyestradiol inhibits hypoxia-inducible factor 1alpha, tumor growth, and angiogenesis and augments paclitaxel efficacy in head and neck squamous cell carcinoma. Clin Cancer Res 24:8665-8673. doi:10.1158/1078-0432.CCR-04-1393

Singh N, Sharma G, Mishra V (2012) Hypoxia inducible factor-1: its potential role in cerebral ischemia. Cell Mol Neurobiol 4:491-507. doi:10.1007/s10571-012-9803-9

Swanson RA, Sharp FR (1994) Infarct measurement methodology. J Cereb Blood Flow Metab 4:697-698. doi:10.1038/jcbfm.1994.88

Wang Q, Peng Y, Chen S, Gou X, Hu B, Du J et al (2009) Pretreatment with electroacupuncture induces rapid tolerance to focal cerebral ischemia through regulation of endocannabinoid system. Stroke 6:2157-2164. doi:10.1161/STROKEAHA.108. 541490

Wang C, Wang Z, Zhang X, Zhang X, Dong L, Xing Y et al (2012a) Protection by silibinin against experimental ischemic stroke: upregulated pAkt, pmTOR, HIF-1alpha and Bcl-2, down-regulated Bax, NF-kappaB expression. Neurosci Lett. 1:45-50. doi:10. 1016/j.neulet.2012.08.078

Wang Q, Wang F, Li X, Yang Q, Li X, Xu N et al (2012b) Electroacupuncture pretreatment attenuates cerebral ischemic injury through alpha7 nicotinic acetylcholine receptor-mediated inhibition of high-mobility group box 1 release in rats. J Neuroinflammation 9:24. doi:10.1186/1742-2094-9-24

Wang F, Gao Z, Li X, Li Y, Li X, Zhong H et al (2013) NDRG2 is involved in anti-apoptosis induced by electroacupuncture pretreatment after focal cerebral ischemia in rats. Neurol Res 4:406-414. doi:10.1179/1743132813Y.0000000159

Wei H, Yao X, Yang L, Wang S, Guo F, Zhou H et al (2014) Glycogen synthase kinase-3beta is involved in electroacupuncture pretreatment via the cannabinoid CB1 receptor in ischemic stroke. Mol Neurobiol 1:326-336. doi:10.1007/s12035-013$8524-5$ 
Wilkins SE, Hyvarinen J, Chicher J, Gorman JJ, Peet DJ, Bilton RL et al (2009) Differences in hydroxylation and binding of Notch and HIF-1alpha demonstrate substrate selectivity for factor inhibiting HIF-1 (FIH-1). Int J Biochem Cell Biol 7:1563-1571. doi:10.1016/j.biocel.2009.01.005

Yang ZH, Sun K, Yan ZH, Suo WH, Fu GH, Lu Y (2010) Panaxynol protects cortical neurons from ischemia-like injury by upregulation of HIF-1alpha expression and inhibition of apoptotic cascade. Chem Biol Interact 1:165-171. doi:10.1016/j.cbi.2009. 09.020

Yang Q, Yan W, Li X, Hou L, Dong H, Wang Q et al (2012) Activation of canonical notch signaling pathway is involved in the ischemic tolerance induced by sevoflurane preconditioning in mice. Anesthesiology 5:996-1005. doi:10.1097/ALN.0b013e31826cb469

Ye Z, Guo Q, Xia P, Wang N, Wang E, Yuan Y (2012) Sevoflurane postconditioning involves an up-regulation of HIF-1alpha and HO-1 expression via PI3 K/Akt pathway in a rat model of focal cerebral ischemia. Brain Res 1463:63-74. doi:10.1016/j.brainres. 2012.04.050

Yuan LB, Dong HL, Zhang HP, Zhao RN, Gong G, Chen XM et al (2011) Neuroprotective effect of orexin-A is mediated by an increase of hypoxia-inducible factor- 1 activity in rat. Anesthesiology 2:340-354. doi:10.1097/ALN.0b013e318206ff6f

Zhang HP, Sun YY, Chen XM, Yuan LB, Su BX, Ma R et al (2014) The neuroprotective effects of isoflurane preconditioning in a murine transient global cerebral ischemia-reperfusion model: the role of the Notch signaling pathway. Neuromolecular Med 1:191-204. doi:10.1007/s12017-013-8273-7

Zhao Y, Chen X, Ma L, Zuo Z, Zhu Z, Zhu X et al (2012) Electroacupuncture pretreatment induces tolerance against focal cerebral ischemia through activation of canonical Notch pathway. BMC Neurosci. doi:10.1186/1471-2202-13-111

Zheng X, Linke S, Dias JM, Zheng X, Gradin K, Wallis TP et al (2008) Interaction with factor inhibiting HIF-1 defines an additional mode of cross-coupling between the Notch and hypoxia signaling pathways. Proc Natl Acad Sci USA 9:3368-3373. doi:10.1073/pnas.0711591105

Zhou D, Matchett GA, Jadhav V, Dach N, Zhang JH (2008) The effect of 2-methoxyestradiol, a HIF-1 alpha inhibitor, in global cerebral ischemia in rats. Neurol Res 3:268-271. doi:10.1179/ $016164107 X 229920$

Zhou H, Zhang Z, Wei H, Wang F, Guo F, Gao Z et al (2013) Activation of STAT3 is involved in neuroprotection by electroacupuncture pretreatment via cannabinoid CB1 receptors in rats. Brain Res 1529:154-164. doi:10.1016/j.brainres.2013.07. 006

Zhu T, Zhan L, Liang D, Hu J, Lu Z, Zhu X et al (2014) Hypoxiainducible factor lalpha mediates neuroprotection of hypoxic postconditioning against global cerebral ischemia. J Neuropathol Exp Neurol 10:975-986. doi:10.1097/NEN.0000000000000118 